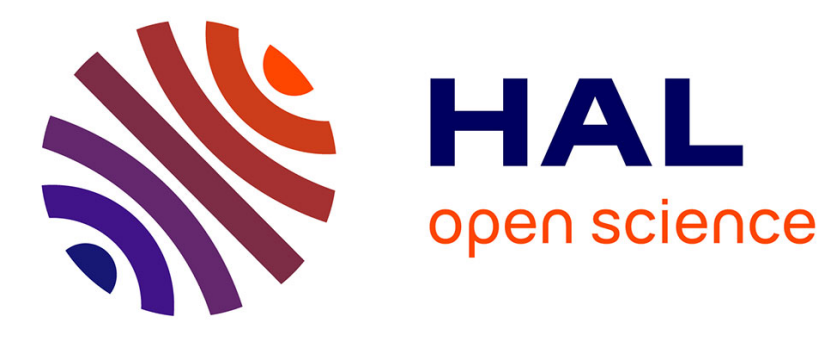

\title{
Influence of Patella Height on Total Knee Arthroplasty: Outcomes and Survival
}

\author{
Romain Gaillard, Christopher Bankhead, Nicolaas Budhiparama, Cécile \\ Batailler, Elvire Servien, Sebastien Lustig
}

\section{To cite this version:}

Romain Gaillard, Christopher Bankhead, Nicolaas Budhiparama, Cécile Batailler, Elvire Servien, et al.. Influence of Patella Height on Total Knee Arthroplasty: Outcomes and Survival. The Journal of Arthroplasty, 2019, 34 (3), pp. 469-477. 10.1016/j.arth.2018.10.037 . hal-02138483

\section{HAL Id: hal-02138483 \\ https://hal.science/hal-02138483}

Submitted on 22 Oct 2021

HAL is a multi-disciplinary open access archive for the deposit and dissemination of scientific research documents, whether they are published or not. The documents may come from teaching and research institutions in France or abroad, or from public or private research centers.
L'archive ouverte pluridisciplinaire HAL, est destinée au dépôt et à la diffusion de documents scientifiques de niveau recherche, publiés ou non, émanant des établissements d'enseignement et de recherche français ou étrangers, des laboratoires publics ou privés.

\section{(ㅇ)(1) $\$$}

Distributed under a Creative Commons Attribution - NonCommerciall 4.0 International 


\title{
Clinical article
}

\section{INFLUENCE OF PATELLA HEIGHT ON TOTAL KNEE ARTHROPLASTY: OUTCOMES AND SURVIVAL}

\author{
Romain GAILLARD ${ }^{\mathrm{a}}$, Christopher BANKHEAD ${ }^{\mathrm{b}}$, Nicolaas BUDHIPARAMA ${ }^{\mathrm{c}}$, Cécile \\ BATAILLER ${ }^{\mathrm{a}}$, Elvire SERVIEN ${ }^{\mathrm{a}}$, Sébastien LUSTIG ${ }^{\mathrm{a}}$
}

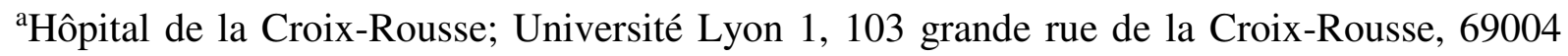
LYON, France

romain.gaillard@chu-lyon.fr

cecile.batailler@chu-lyon.fr

elvire.servien@chu-lyon.fr

sebastien.lustig@gmail.com

${ }^{b}$ Department of Orthopaedics, University of New Mexico; 1 University of New Mexico, MSC 10 5600, ALBUQUERQUE, NM 87106, USA

cbankhead@salud.unm.edu

'Nicolaas Institute of Constructive Orthopaedics Research and Education Foundation for Arthroplasty \& Sports Medicine, Medistra Hospital, Jl. Jend. Gatot Subroto Kav. 59, Jakarta, 12950 Indonesia

ncbjr@yahoo.com

\section{Corresponding author:}

Sébastien LUSTIG

Hôpital de la Croix-Rousse; Université Lyon 1,

103 grande rue de la Croix-Rousse,

69004 LYON, France

sebastien.lustig@gmail.com 
1 INFLUENCE OF PATELLA HEIGHT ON TOTAL KNEE ARTHROPLASTY:

2 OUTCOMES AND SURVIVAL

3

4 ABSTRACT

5

6

\section{BACKGROUND}

7

To assess clinical results of total knee arthroplasty (TKA) with pre-operative patella alta or

8 patella baja, compared to TKA with pre-operative normal patellar height.

9

10

\section{METHODS}

Patella height was measured using the Blackburne-Peel (BP) method in an initial, prospective cohort of 4103 TKAs performed at a single center. Three groups were defined: normal patella height $(0.54<\mathrm{BP}$ index $\leq 1.06)$, patella alta $(\mathrm{BP}$ index $>1.06)$ and patella baja (BP index $\leq 0.54)$. Pre-operative and post-operative clinical and radiological characteristics were collected and compared. All prostheses used a system of posterior stabilization by a third condyle. The primary outcome measure was the post-operative Knee Society Score (KSS). Statistical analysis was performed using Student t-test and Chi-2 test, with $\mathrm{p}<0.05$.

\section{RESULTS}

223 TKAs with pre-operative patella alta, 307 TKAs with pre-operative patella baja and 2248 with pre-operative normal patella height, mean follow-up 39.3 months (range 24-239), were ultimately included. Post-operatively, there was no difference between the three groups in terms of KSS (knee and function scores). Patella baja statistically decreased the maximal flexion $\left(118.2^{\circ}\right.$ vs $\left.115.5^{\circ} ; p=0.002\right)$ and increased the rate of patellar fracture $(0.9 \%$ vs $2 \%$; $p=0.05)$. Finally, survival rates were similar in the three groups with each above $91 \%(+/-$ $2 \%)$ at 10 years.

CONCLUSION

29 Clinical and radiological results for TKAs with pre-operative patella alta and patella baja were comparable to TKAs with a normal pre-operative patellar height. Risk of post-operative patellar fracture increased for patients with pre-operative patella baja. 
32 KEYWORDS: Total knee arthroplasty, patella alta, patella baja, KSS, Blackburne-Peel index 33

34 CLINICAL RELEVANCE: Equivalent clinical and radiological results between TKAs with 35 pre-operative patella alta or baja and TKA with a pre-operative normal patellar height.

36 Original subject never explored in the literature.

37

38

39

40

41 ABBREVIATIONS:

42 BP: Blackburne-Peel index

43 BMI: Body Mass Index

44 DFO: Distal Femoral Osteotomy

45 HKA: Hip Knee Ankle

46 HTO: High Tibial Osteotomy

47 KSS: Knee Society Score

48 mFA: mechanical Femoral Axis

49 mFTA: mechanical Femoro-Tibial Axis

50 mTA: mechanical Tibial Axis

51 TKA: Total Knee Arthroplasty 


\section{INTRODUCTION}

53 tendon[4-6]. arthritis $[10,11]$. malpositioning[5,16].

Variation of the patellar height in the general population is not uncommon. On the one hand, patella alta may be asymptomatic and incidentally discovered, or it may be part of patello-femoral instability syndrome[1-3]. On the other hand, patella baja is frequently secondary to a previous surgery or trauma about the knee with a shortening of the patellar

Patella alta causes, from a biomechanical point of view, a migration of the contact point of the patello-femoral joint near the apex of the patella, which in turn causes a decrease in the effective lever arm of the extensor mechanism[7], an increase in transmitted patellofemoral forces[8,9], and ultimately the appearance of premature inferior patello-femoral

Each of these biomechanical elements which are detrimental to the native knee can also occur in the prosthetic knee, potentially leading to post-operative patello-femoral complications such as instability or patella button loosening[12,13].

Patella baja in the native knee is responsible for pain and limited flexion, due to the distalisation of the patella in the femoral groove[4,14]. Biomechanically, this causes a superior migration of the contact point on the patella with a decrease of the patellar height[15], leading to a risk of early patello-femoral osteoarthritis.

The induced stiffness can result in a difficult approach to the knee for prosthesis implantation, with a risk of patellar fracture, extensor mechanism lesion and implant

There are currently few studies which report the results of total knee arthroplasty (TKA) with patellar resurfacing performed in pre-operative patella alta or baja, or the possible post-operative patello-femoral complications that could result.

The objective of this study was to compare the clinical and radiographic results of two cohorts of TKA patients with pre-operative patella alta and pre-operative patella baja with a group of patients having normal pre-operative patellar height. The post-operative complications as well as the implant survival of each of these three populations were analyzed and compared. We hypothesized that there would be no clinical or radiographic difference 
between the three groups and that the post-operative patello-femoral complication rate would be similar.

\section{MATERIALS AND METHODS}

\section{Study population}

Between November 1987 and March 2015 we prospectively followed all primary TKAs performed at a single center by 16 senior surgeons using the same surgical technique. Patients were followed clinically and radiographically after surgery first at 2 months, then 1 year, and then every 2 years afterwards. This series consists of 4103 primary TKAs.

Patellar height was measured on each pre- and post-operative lateral radiograph, using the Blackburne-Peel (BP) index[17] (Fig. 1). All radiographs were performed in our institution with a standardized method, increasing reproducibility.

The measurement was performed on a lateral radiograph, with $30^{\circ}$ of flexion according to the same established radiological protocol. The measurements were initially made by a junior surgeon (resident) and then independently verified by a senior surgeon. If there was a discrepancy greater than $5 \%$, a new measurement was independently performed by a second senior surgeon. Freehand measurements on acetate films were performed before 2002, while digital computer software with numeric measurements was used after 2002.

For the measurement of the BP index on the native knee, the joint line was defined on the lateral radiograph by the tangent to the upper edge of the tibial plateau. The patellar measurement consisted of only the articular portion of the patella.

For measurement of the BP index on TKA, the joint line was defined on the lateral radiograph by the tangent to the upper edge of the tibial polyethylene. The patellar measurement consisted of only the patellar button.

This index made it possible to define 3 separate pre-operative groups, according to the standards defined by Blackburne and Peel[17]:

- Normal patellar height with a BP index =0.54-1.06 (N=3301) (Fig. 2);

- Patella alta with a BP index > $1.06(\mathrm{~N}=330)($ Fig. 3);

- Patella baja with a BP index <0.54 (N=472) (Fig. 4). 
Excluded from the study due to lack of follow up were knees having clinical and

122

123

124

125

126

127

128

129

130

131

132

133

134

135

136

137

138

139

140

141

142

143

144

145

146

147

148

149

150

151

152 radiographic follow-up less than 24 months, which consisted of knees lost to follow-up $(\mathrm{N}=398)$ and knees operated on after March 2013 (N=927). In total, a cohort of 2248 knees were included for pre- and post-operative analysis of TKA with normal pre-operative patellar height, and 223 were included in the cohort of TKA with pre-operative patella alta, and 307 were included in the cohort of TKA with pre-operative patella baja (Fig. 5), all with an average follow-up of 39.3 months (24-239).

All operative technical data including the operative reports as well as pre- and postoperative clinical and radiographic data were prospectively collected in a computer database created in 1995 (data from TKA's performed between 1987 and 1995 were entered in the database retrospectively).

Clinical follow-up was performed using the Knee Society Score (KSS) questionnaire to calculate the corresponding KSS score at each follow-up visit[18]. The Knee Society radiographic evaluation consisted of an anteroposterior and lateral view of the operative knee, a skyline view of the patella at $45^{\circ}$ of knee flexion, as well as a standing bilateral hip to ankle radiograph to assess mechanical axis[19]. All local and systemic complications resulting from the surgery were noted and recorded in the database, according to the standardized list and definitions of the Knee Society[20].

The primary outcome of this study was the mean post-operative KSS score at the last follow-up. The secondary outcomes were the mean maximal flexion, the patello-femoral complications, and the survival of the different cohorts.

The Advisory Committee on the Treatment of Health Research Information (CCTIRS) approved this study on January 24, 2012 and on March 9, 2015 under the identification number 11-681.

\section{Operative technique}

Before 1996, the medial approach was systematically used. After 1996, the choice of approach became a function of the pre-operative deformity: medial for genu varum, lateral for 
153 genu valgum, and with tibial tubercle osteotomy if necessary (128 patients in this series for 154 stiffness and/or difficult lateral approach for valgus[21]).

155 The surgical technique used a posterior reference, beginning with the tibial cut and 156 followed by the posterior femoral cut (balancing is performed in flexion then checked in 157 extension and balanced with a distractor before the distal femoral cut is performed). All 158 patellae were resurfaced. All of the implants were cemented, except for 132 femoral 159 components (3.2\%) which were coated with hydroxyapatite and had been the subject of 160 another prospective study published in 2011[22].

161 Since 1987, all of the implants have been designed and fabricated by the same 162 manufacturer (Wright-Tornier-Corin ${ }^{\circledR}, 38330$ Montbonnot Saint Martin, FRANCE). All of 163 the implants have a similar design, using posterior stabilization through a third condyle (HLS 164 family implants).

Statistical analysis

Statistical data was analyzed using the chi2 test for comparison of distributions 169 between the two groups and the Student's t-test for quantitative values (large and normal-law variables), using $\mathrm{R} \odot$ software ( $\mathrm{R}$ Foundation for Statistical Computing, http://www.R-

171 project.org). The Kaplan-Meier survival curves were made and compared using a LogRank 172 test with the help of the Technical University of Denmark's online site 173 (https://www.statcom.dk). The results were considered significant if $\mathrm{p}<0.05$.

RESULTS

Pre-operative (Table 1)

The rate of lateral tibio-femoral arthritis was statistically greater in the cohort with pre-operative patella alta $(p=0.04)$ compared to the cohort with normal pre-operative patellar height, while there was an increased incidence of medial tibio-femoral arthritis in the cohort with pre-operative patella baja $(p=0.02)$.

The average maximum flexion was statistically greater in the cohort with preoperative patella alta $(p=0.004)$ compared to the cohort with normal pre-operative patellar 
height, while there was statistically decreased flexion in the cohort with pre-operative patella baja $(p=0.006)$.

The mean KSS Knee and Function scores were statistically greater in the cohort with pre-operative patella alta $(p=0.003$ and $p=0.02)$ compared to the cohort with normal preoperative patellar height, while there were statistically lower scores in the cohort with preoperative patella baja $(p<0.001$ and $p=0.03)$.

\section{Post-operative (Table 2)}

No statistically significant difference was found in the selected clinical and radiographic parameters between the normal pre-operative patellar height group and the patella alta group.

A statistically significant difference was found between the normal pre-operative patellar height group and the patella baja group for the mean post-operative maximal flexion $\left(118.2^{\circ}\right.$ vs $\left.115.5^{\circ} ; p=0.002\right)$ and the mean post-operative mFTA (mechanical Femoro-Tibial Axis $)\left(178.9^{\circ}\right.$ vs $\left.178.2^{\circ} ; p=0.006\right)$.

\section{Patello-femoral complications (Table 3)}

No statistically significant difference was found in the rates of complication between the different groups in terms of stiffness, clunk syndrome, extensor mechanism rupture, patella button loosening and patello-femoral instability. A statistically significant difference was found for patella fracture between patella baja and normal patellar height cohorts, with an increased rate of patella frature in patella baja group $(0.9 \%$ vs $2 \% ; p=0.05)$.

\section{Survival analysis}

Figure 6 demonstrates the survival curves of TKA with normal pre-operative patellar height $(98 \%+/-1 \%$ at 5 years), TKA with pre-operative patella alta $(96 \%+/-4 \%$ at 5 years) and TKA with pre-operative patella baja ( $98 \%+/-4 \%$ at 5 years). An event was defined as the revision of at least one prosthetic component, patella button and polyethylene insert excluded. No statistical difference was found between the three groups $(p=0.6)$. 
From the cohort of the patients with normal pre-operative patellar height (Table 4), no statistically significant difference was found if patients had post-operative patella alta or baja, compared to the cohort with normal post-operative patellar height.

From the cohort of the patients with pre-operative patella alta (Table 5), no statistically significant difference was found if patients had post-operative patella alta, compared to the cohort with normal post-operative patellar height. A statistically significant difference was found between the normal post-operative patellar height group and the post-operative patella baja group for the mean post-operative KSS knee score ( 89.3 vs $84.8 ; p=0.02)$.

From the cohort of the patients with pre-operative patella baja (Table 6), no statistically significant difference was found if patients had post-operative patella alta, compared to the cohort with normal post-operative patellar height. A statistically significant difference was found between the normal post-operative patellar height group and the postoperative patella baja group for the rate of post-operative stiffness $(1.9 \%$ vs $5.5 \% ; p=0.03)$.

No correlation was found between the level of BP index for patients with preoperative patella baja and the KSS knee and function scores at the end of the follow-up

Specific results of patients with tibial tubercle osteotomy (Table 7)

For patients with pre-operative patella baja, stiffness is statistically more responsible for tibial tubercle osteotomy than for patients with normal pre-operative patellar height $(33.3 \%$ vs $10.9 \% ; p=0.02)$.

The risk of post-operative patella baja after TKA with tibial tubercle osteotomy is statistically higher than for patients with normal pre-operative patellar height $(46.7 \% \mathrm{vs}$ $28.7 \% ; p=0.03)$.

\section{DISCUSSION}

The main contribution of this study is to demonstrate there is no significant difference in post-operative KSS scores, patient satisfaction or in survival rates of TKA performed with normal pre-operative patellar height. However, in patients with pre-operative patella baja, it 
253 demonstrates a correlated decrease in post-operative flexion and an increased risk of patellar 254 fracture post-operatively.

The concept of patella alta/baja is correlated with the measurement index that is used.

257 A number of methods to measure the height of the patella exist (Blackburne-Peel, Caton258 Deschamps, Insall-Salvati, Labelle-Laurin, etc.). The choice to use the Blackburne-Peel 259 index[17], described in 1977, was motivated by its excellent inter- and intra-observer 260 reproducibility as demonstrated by Berg et al.[23] in 1996, as well as Seil et al.[24] in 2000. 261 Additionally, this index seems to be the best able to objectively measure patella height in a prosthetic knee. On the other hand it is essential to standardize the knee position at $30^{\circ}$ of flexion during the lateral radiograph in order to avoid a significant variation in the measurements that could distort the values of the index[25].

Patella alta provides relatively favorable pre-operative clinical conditions such as a significantly increased degree of flexion compared with knees having normal pre-operative patellar height $\left(120.5^{\circ}\right.$ vs $\left.117^{\circ}\right)$, as well as better overall KSS scores. In contrast, cases of patella baja are significantly more likely to present with pre-operative stiffness, usually related to patella tendon retraction secondary to trauma[6,26] or previous surgery[27,28].

Our study found a significant increase in lower limb valgus alignment in the cohort of 273 knees with patella alta, corroborating recent findings[29] that show a significant link between 274 patella instability (often associated with patella alta) and lower limb valgus. Conversely, we discovered a majority of lower limb varus alignment in the cohort of knees with patella baja.

Paradoxically, we found a significant decrease in the rate of patello-femoral arthritis in 277 the patellae alta and baja cohorts compared to the knees with normal patellar height. This 278 finding is at odds with previous studies showing a change in patello-femoral contact forces in 279 cases of patellae alta or baja which are responsible for premature arthritis of the 280 patella[9,11,15]. This observed inconsistency seems to be related to the method used to assess 281 patello-femoral arthritis in our series, which takes into account only an axial patellar view and cannot detect more inferior patello-femoral arthritis.

Our study found no significant difference in post-operative KSS scores, patient 285 satisfaction or in survival rates of TKA performed with pre-operative patella alta or patella 
baja when compared to TKA performed on knees having a normal pre-operative patellar 287 height.

However, there exists a correlated decrease in post-operative flexion for patients with pre-operative patella baja and an increased risk of patellar fracture post-operatively. These results corroborate with the studies from Singerman et al.[15] and Chonko et al.[5] that showed a correlation between patella baja and modification of the patella-femoral contact forces, responsible for decreasing range of motion and increasing rate of patellar fracture.

It has also been shown several times there is a moderate decrease in patellar height following TKA, called pseudo patella baja[30], which is related to both the management of the distal femoral cut[5,31], and also a decrease in the length of the patella tendon[32].

It can explain the normalization of the mean post-operative Blackburne-Peel index in the cohort with pre-operative patella alta (0.67 post-operative vs 1.32 pre-operative). Therefore, it is quite logical to deduce that the pathological biomechanics secondary to patella alta are also normalized after TKA, thus producing no negative consequences on the clinical or radiographic results of the implants.

We need to moderate this purpose for patients with persistent post-operative patella baja, or important decrease of patellar height for patients with pre-operative patella alta. The post-operative results consist in an increase rate of post-operative stiffness in the first case, and a decrease of the mean KSS knee score in the second case.

Thus, the presence of pre-operative patella alta does not appear to be responsible for 308 systematic post-operative patella alta, which can lead to patella button mechanical stress or cause patella instability. It also does not affect implant survival after TKA when compared to the normal patellar height.

Conversely, pre-operative patella baja seems to be a major risk for post-operative patellar fracture and stiffness, especially if there is no correction of the patellar height during the surgery. In these cases, a surgeon may elect to avoid patella resurfacing, or to combine the TKA implantation with a proximalization of the anterior tibial tuberosity[14].

Tibial tubercle osteotomy was often combined with lateral approach for severe valgus before the 2000s, as described by Keblish[21]. Then it was reserved for knees with severe stiffness (flexion $<90^{\circ}$ ) to help with exposure of the joint, especially in patients with preoperative patella baja. Otherwise, tibial tubercle osteotomy in patients with pre-operative 
patella baja increased the risk of keeping a post-operative patella baja secondary to potential inflammation and shortening of the patellar tendon[32].

Our study had several limitations. Despite regular monitoring, those lost to follow-up remain numerous (10\% of the initial cohort). This deficiency biases our results. Additionally, the mean duration to latest follow-up remains short (3.3 years), introducing a positive bias toward long-term complications and survival analysis. In addition, there is a great variability in the duration of follow-up of patients (from 24 to 239 months), and only the clinical score at the last follow-up was taken into account in our study.

Despite the repeated and validated measurements of the BP index, we could not calculate the inter-observer reliability coefficient because the data pertaining to the various measurements is not present in the database. Nevertheless, due to the large size of each population analyzed, the power of our study is quite satisfactory.

Moreover, given the uniformity of our initial cohort used, we can reasonably conclude there is no negative impact of pre-operative patella alta or baja on clinical and radiographic results when performing TKA, especially if the management of the patellar height during the surgery is performed to avoid post-operative patella baja.

\section{CONCLUSION}

This study highlights the absence of any significant difference in terms of postoperative KSS scores, patient satisfaction or survival results in TKA performed with preoperative patella alta or baja compared to TKA performed on knees with normal pre-operative patellar height. However, an increased risk of patellar fracture is correlated with pre-operative patella baja. This result invites to reconsider the systematic patella resurfacing in patients with pre-operative patella baja. 
347 COMPLIANCE WITH ETHICAL STANDARDS.

348

349

- Conflict of Interest:

350 No benefits in any form have been received or will be received from a commercial party 351 related directly or indirectly to the subject of this article.

352

353 - Funding:

354 This research did not receive any specific grant from funding agencies in the public, 355 commercial, or not-for-profit sectors. 
358 [1] Geenen E, Molenaers G, Martens M. Patella alta in patellofemoral instability. Acta 359 Orthop Belg 1989;55:387-93.

360 [2] Møller BN, Krebs B, Jurik AG. Patellar height and patellofemoral congruence. Arch 361 Orthop Trauma Surg Arch Für Orthop Unf-Chir 1986;104:380-1.

362 [3] Dejour H, Walch G, Nove-Josserand L, Guier C. Factors of patellar instability: an 363 anatomic radiographic study. Knee Surg Sports Traumatol Arthrosc Off J ESSKA 1994;2:19_ 36426.

365 [4] Caton J, Deschamps G, Chambat P, Lerat JL, Dejour H. [Patella infera. Apropos of 366128 cases]. Rev Chir Orthopédique Réparatrice Appar Mot 1982;68:317-25.

367 [5] Chonko DJ, Lombardi AV, Berend KR. Patella baja and total knee arthroplasty 368 (TKA): etiology, diagnosis, and management. Surg Technol Int 2004;12:231-8.

369 [6] Mariani PP, Del Signore S, Perugia L. Early development of patella infera after knee 370 fractures. Knee Surg Sports Traumatol Arthrosc Off J ESSKA 1994;2:166-9.

371 [7] Ward SR, Terk MR, Powers CM. Influence of patella alta on knee extensor mechanics. 372 J Biomech 2005;38:2415-22. doi:10.1016/j.jbiomech.2004.10.010.

373 [8] Luyckx T, Didden K, Vandenneucker H, Labey L, Innocenti B, Bellemans J. Is there a 374 biomechanical explanation for anterior knee pain in patients with patella alta?: influence of 375 patellar height on patellofemoral contact force, contact area and contact pressure. J Bone Joint 376 Surg Br 2009;91:344-50. doi:10.1302/0301-620X.91B3.21592.

377 [9] Ward SR, Powers CM. The influence of patella alta on patellofemoral joint stress 378 during normal and fast walking. Clin Biomech Bristol Avon 2004;19:1040-7. 379 doi:10.1016/j.clinbiomech.2004.07.009.

380 [10] Ahlbäck S, Mattsson S. Patella alta and gonarthrosis. Acta Radiol Diagn (Stockh) 381 1978;19:578-84.

382 [11] Stefanik JJ, Zhu Y, Zumwalt AC, Gross KD, Clancy M, Lynch JA, et al. Association 383 between patella alta and the prevalence and worsening of structural features of patellofemoral 384 joint osteoarthritis: the multicenter osteoarthritis study. Arthritis Care Res 2010;62:1258-65. 385 doi:10.1002/acr.20214.

386 [12] Briard JL, Hungerford DS. Patellofemoral instability in total knee arthroplasty. J 387 Arthroplasty 1989;4 Suppl:S87-97.

388 [13] Schiavone Panni A, Cerciello S, Del Regno C, Felici A, Vasso M. Patellar resurfacing 389 complications in total knee arthroplasty. Int Orthop 2014;38:313-7. doi:10.1007/s00264-013- 
2244-3.

[14] Drexler M, Dwyer T, Marmor M, Sternheim A, Cameron HU, Cameron JC. The treatment of acquired patella baja with proximalize the tibial tuberosity. Knee Surg Sports Traumatol Arthrosc Off J ESSKA 2013;21:2578-83. doi:10.1007/s00167-012-2278-x.

[15] Singerman R, Davy DT, Goldberg VM. Effects of patella alta and patella infera on patellofemoral contact forces. J Biomech 1994;27:1059-65.

[16] Kim J, Nelson CL, Lotke PA. Stiffness after total knee arthroplasty. Prevalence of the complication and outcomes of revision. J Bone Joint Surg Am 2004;86-A:1479-84.

[17] Blackburne JS, Peel TE. A new method of measuring patellar height. J Bone Joint Surg Br 1977;59:241-2.

[18] Lingard EA, Katz JN, Wright RJ, Wright EA, Sledge CB, Kinemax Outcomes Group. Validity and responsiveness of the Knee Society Clinical Rating System in comparison with the SF-36 and WOMAC. J Bone Joint Surg Am 2001;83-A:1856-64.

[19] Ewald FC. The Knee Society total knee arthroplasty roentgenographic evaluation and scoring system. Clin Orthop 1989:9-12.

[20] Healy WL, Della Valle CJ, Iorio R, Berend KR, Cushner FD, Dalury DF, et al. Complications of total knee arthroplasty: standardized list and definitions of the Knee Society. Clin Orthop 2013;471:215-20. doi:10.1007/s11999-012-2489-y.

[21] Keblish PA. The lateral approach to the valgus knee. Surgical technique and analysis of 53 cases with over two-year follow-up evaluation. Clin Orthop 1991:52-62.

[22] Demey G, Servien E, Lustig S, Aï Si Selmi T, Neyret P. Cemented versus uncemented femoral components in total knee arthroplasty. Knee Surg Sports Traumatol Arthrosc Off J ESSKA 2011;19:1053-9. doi:10.1007/s00167-010-1347-2.

[23] Berg EE, Mason SL, Lucas MJ. Patellar height ratios. A comparison of four measurement methods. Am J Sports Med 1996;24:218-21.

[24] Seil R, Müller B, Georg T, Kohn D, Rupp S. Reliability and interobserver variability in radiological patellar height ratios. Knee Surg Sports Traumatol Arthrosc Off J ESSKA 2000;8:231-6. doi:10.1007/s001670000121.

[25] Narkbunnam R, Chareancholvanich K. Effect of patient position on measurement of patellar height ratio. Arch Orthop Trauma Surg 2015;135:1151-6. doi:10.1007/s00402-0152268-9.

[26] Jiang X, Zhang Y, Liu J. Patella infera following patellar tendon contracture after closed trauma. Chin Med J (Engl) 2013;126:3990-1.

[27] Kaper BP, Bourne RB, Rorabeck CH, Macdonald SJ. Patellar infera after high tibial 
424 osteotomy. J Arthroplasty 2001;16:168-73. doi:10.1054/arth.2001.20538.

425 [28] Gokay NS, Erginer R, Dervisoglu S, Yalcin MB, Gokce A. Patella infera or patellar 426 tendon adherence after high tibial osteotomy. Knee Surg Sports Traumatol Arthrosc Off J 427 ESSKA 2014;22:1591-8. doi:10.1007/s00167-012-2293-y.

428 [29] Hermans K, Claes S, Bellemans J. Valgus instability as a cause for recurrent lateral 429 patellar dislocation: a new mechanism for patellofemoral instability? Acta Orthop Belg $430 \quad 2013 ; 79: 495-501$.

431 [30] Kazemi SM, Daftari Besheli L, Eajazi A, Miniator Sajadi MR, Okhovatpoor MA, 432 Farhang Zanganeh R, et al. Pseudo-Patella Baja after total knee arthroplasty. Med Sci Monit 433 2011;17:CR292-6. doi:10.12659/MSM.881770.

434 [31] Khakharia S, Scuderi GR. Restoration of the distal femur impacts patellar height in 435 revision TKA. Clin Orthop 2012;470:205-10. doi:10.1007/s11999-011-1995-7.

436 [32] Weale AE, Murray DW, Newman JH, Ackroyd CE. The length of the patellar tendon 437 after unicompartmental and total knee replacement. J Bone Joint Surg Br 1999;81:790-5. 
442 Table 1 Pre-operative clinical and radiological characteristics

\begin{tabular}{|c|c|c|c|}
\hline & Normal $(\mathrm{N}=2248)$ & Alta $(\mathrm{N}=223)$ & Baja $(\mathrm{N}=307)$ \\
\hline Mean pre-operative BP index & $0.77[0.54-1.06]$ & $1.32[1.07-4.4] *$ & $0.45[0.06-0.53]^{*}$ \\
\hline Mean age & 70.7 years [26-92] & 70.9 years [28-91] & 69.4 years [26-91] \\
\hline Mean BMI & $29 \mathrm{~kg} / \mathrm{m}^{2}[15-52]$ & $28.8 \mathrm{~kg} / \mathrm{m}^{2}[18-49]$ & $28.7 \mathrm{~kg} / \mathrm{m}^{2}[16-51]$ \\
\hline \multicolumn{4}{|l|}{ Sex } \\
\hline Male & $639(28 \%)$ & $76(34 \%) *$ & $93(30 \%)$ \\
\hline Female & $1609(72 \%)$ & $147(66 \%) *$ & $147(70 \%)$ \\
\hline \multicolumn{4}{|l|}{ Previous surgery } \\
\hline Closing HTO & $25(1.1 \%)$ & $4(1.8 \%)$ & $3(1 \%)$ \\
\hline Opening HTO & $134(6 \%)$ & $9(4 \%)$ & $19(5.9 \%)$ \\
\hline Opening DFO & $8(0.4 \%)$ & $1(0.5 \%)$ & 0 \\
\hline Tibial tubercle transfer & $32(1.4 \%)$ & $3(1.4 \%)$ & $5(1.6 \%)$ \\
\hline Osteosynthesis & $30(1.3 \%)$ & $6(2.7 \%)$ & $6(2 \%)$ \\
\hline Arthroscopic arthrolysis & $4(0.2 \%)$ & 0 & $3(1 \%) *$ \\
\hline \multicolumn{4}{|l|}{ Type of arthritis } \\
\hline Medial compartment & $1540(68.5 \%)$ & $131(58.7 \%) *$ & $246(80.1 \%) *$ \\
\hline Lateral compartment & $348(15.5 \%)$ & $48(21.5 \%) *$ & $26(8.5 \%) *$ \\
\hline Global & $35(1.6 \%)$ & $3(1.4 \%)$ & $8(2.6 \%)$ \\
\hline Patello-femoral compartment & $92(4.1 \%)$ & $11(4.9 \%)$ & $2(0.7 \%) *$ \\
\hline$N C$ & $233(10.3 \%)$ & $30(13.5 \%) *$ & $25(8.1 \%)$ \\
\hline \multicolumn{4}{|l|}{ Range of motion } \\
\hline Mean flexion contracture & $3.73^{\circ}[0-30]$ & $2.94^{\circ}[0-45]$ & $4.3^{\circ}[0-30]$ \\
\hline Mean maximal flexion & $117^{\circ}[35-140]$ & $120.5^{\circ}[40-140] *$ & $113.1^{\circ}[30-140]^{*}$ \\
\hline \multicolumn{4}{|l|}{ Mean KSS score } \\
\hline Knee & $49.6[0-100]$ & $53.3[3-97] *$ & $44.2[0-100] *$ \\
\hline Function & $57.5[0-100]$ & $60.7[0-100] *$ & $55.4[0-100]$ \\
\hline \multicolumn{4}{|l|}{\begin{tabular}{|l} 
Long leg films \\
\end{tabular}} \\
\hline Mean $m F T A$ & $176,2^{\circ}[155-207]$ & $176.7^{\circ}[158-201]$ & $173.4^{\circ}[156-202] *$ \\
\hline Mean $m F A$ & $91,3^{\circ}[73-110]$ & $91.4^{\circ}[81-104]$ & $90.6^{\circ}[79-118] *$ \\
\hline Mean $m T A$ & $87^{\circ}[69-106]$ & $87.2^{\circ}[70-103]$ & $85.9^{\circ}[74-100] *$ \\
\hline \multicolumn{4}{|l|}{ Patello-femoral arthritis } \\
\hline Normal & $502(22.3 \%)$ & $76(34.1 \%) *$ & $43(14 \%) *$ \\
\hline Remodeling & $749(33.3 \%)$ & $57(25.6 \%) *$ & $95(30.9 \%) *$ \\
\hline Medial side & $204(9.1 \%)$ & $18(8.1 \%)$ & $37(12.1 \%) *$ \\
\hline
\end{tabular}




\begin{tabular}{|c|c|c|c|}
\hline Lateral side & $342(15.2 \%)$ & $35(15.7 \%)$ & $43(14 \%)$ \\
\hline Global & $426(19 \%)$ & $36(15.7 \%) *$ & $88(28.7 \%) *$ \\
\hline Chondrocalcinosis & $14(0.6 \%)$ & $2(0.8 \%)$ & $1(0.3 \%)$ \\
\hline$N C$ & $11(0.5 \%)$ & 0 & 0 \\
\hline \multicolumn{4}{|l|}{ Patello-femoral positioning } \\
\hline Medial dislocation & $18(0.8 \%)$ & $1(0.4 \%)$ & $1(0.3 \%)$ \\
\hline Lateral subluxation & $286(12.7 \%)$ & $24(10.8 \%)$ & $32(10.4 \%)$ \\
\hline Centered & $1919(85.4 \%)$ & $195(87.4 \%)$ & $271(88.3 \%)$ \\
\hline Medial subluxation & $16(0.7 \%)$ & $2(1 \%)$ & $3(1 \%)$ \\
\hline$N C$ & $9(0.5 \%)$ & $1(0.4 \%)$ & 0 \\
\hline
\end{tabular}

444

445

446

447

448

449

450

451

452

BP index: Blackburne-Peel index; BMI: Body Mass Index; DFO: Distal Femoral Osteotomy; HTO: High Tibial Osteotomy; KSS: Knee Society Score; mFA: mechanical Femoral Axis; mFTA: mechanical Femoro-Tibial Axis; mTA: mechanical Tibial Axis

* significant difference $(\mathrm{p}<0.05)$ comparing the normal group

Table 2 Post-operative clinical and radiological characteristics

\begin{tabular}{|c|c|c|c|}
\hline & Normal $(N=2248)$ & Alta $(\mathrm{N}=223)$ & Baja $(\mathrm{N}=307)$ \\
\hline Mean follow-up (months) & 40.1 [24-239] & 34.4 [24-138] & 37.1 [24-179] \\
\hline Mean post-operative BP index & $0.64[0.1-2]$ & $0.67[0.1-1.36] *$ & $0.56[0.1-1.16] *$ \\
\hline \multicolumn{4}{|l|}{ Range of motion } \\
\hline Mean flexion contracture & $0.5^{\circ}[0-25]$ & $0.5^{\circ}[0-15]$ & $0.6^{\circ}[0-15]$ \\
\hline Mean maximal flexion & $118.2^{\circ}[60-140]$ & $118.7^{\circ}[70-140]$ & $115.5^{\circ}[70-140] *$ \\
\hline \multicolumn{4}{|l|}{ Mean KSS score } \\
\hline Knee & 88.3 [24-100] & $88.1[42-100]$ & $87.6[33-100]$ \\
\hline Function & $77.9[0-100]$ & 80.4 [20-100] & $78.2[0-100]$ \\
\hline \multicolumn{4}{|l|}{ Satisfaction } \\
\hline Very good and good & $2067(92 \%)$ & $204(91.5 \%)$ & $285(92.8 \%)$ \\
\hline Bad and very bad & $158(7 \%)$ & $18(7.7 \%)$ & $20(6.5 \%)$ \\
\hline$N C$ & $23(1 \%)$ & $1(0.8 \%)$ & $2(0.7 \%)$ \\
\hline \multicolumn{4}{|l|}{ Long leg films } \\
\hline Mean mFTA & $178.9^{\circ}[170-188]$ & $178.8^{\circ}[171-186]$ & $178.2^{\circ}[168-192] *$ \\
\hline Mean $m F A$ & $89.5^{\circ}[80-98]$ & $89.6^{\circ}[82-95]$ & $89.1^{\circ}[81-95] *$ \\
\hline Mean $m T A$ & $89.5^{\circ}[80-96]$ & $89.5^{\circ}[85-96]$ & $89.2^{\circ}[76-98]$ \\
\hline \multicolumn{4}{|l|}{$\begin{array}{l}\text { Sagittal positioning of patella } \\
\text { button }\end{array}$} \\
\hline High & $137(6.1 \%)$ & $11(4.9 \%)$ & $18(5.9 \%)$ \\
\hline
\end{tabular}




\begin{tabular}{|c|c|c|c|}
\hline $\begin{array}{r}\text { Centered } \\
\text { Low } \\
N C\end{array}$ & $\begin{array}{c}1923(85.5 \%) \\
10(0.5 \%) \\
178(7.9 \%)\end{array}$ & $\begin{array}{c}198(88.8 \%) \\
0 \\
14(6.3 \%)\end{array}$ & $\begin{array}{c}266(86.6 \%) \\
0 \\
23(7.5 \%)\end{array}$ \\
\hline \multicolumn{4}{|l|}{$\begin{array}{l}\text { Coronal positioning of patella } \\
\text { button }\end{array}$} \\
\hline Lateral & $18(0.8 \%)$ & 0 & $2(0.7 \%)$ \\
\hline Centered & $2028(90.2 \%)$ & $207(92.8 \%)$ & $280(91.1 \%)$ \\
\hline Medial & $20(0.9 \%)$ & $2(0.9 \%)$ & $2(0.7 \%)$ \\
\hline$N C$ & $182(8.1 \%)$ & $14(6.3 \%)$ & $23(7.5 \%)$ \\
\hline \multicolumn{4}{|l|}{ Patello-femoral positioning } \\
\hline Medial dislocation & $5(0.2 \%)$ & 0 & 0 \\
\hline Lateral subluxation & $122(5.5 \%)$ & $16(7.2 \%)$ & $17(5.5 \%)$ \\
\hline Centered & $1956(87 \%)$ & $192(86.1 \%)$ & $270(88 \%)$ \\
\hline Medial subluxation & $3(0.1 \%)$ & 0 & $1(0.3 \%)$ \\
\hline$N C$ & $162(7.2 \%)$ & $15(6.7 \%)$ & $19(6.2 \%)$ \\
\hline
\end{tabular}

453

454

455

456

457

458

459

460

461

\begin{tabular}{|c|c|c|c|}
\hline & Normal $(N=2248)$ & Alta $(\mathrm{N}=223)$ & Baja $(N=307)$ \\
\hline Stiffness & $48(2.1 \%)$ & $2(0.9 \%)$ & $11(3.6 \%)$ \\
\hline Clunk syndrome & $18(0.8 \%)$ & $2(0.9 \%)$ & $4(1.3 \%)$ \\
\hline Extensor mechanism rupture & $4(0.2 \%)$ & 0 & 0 \\
\hline Patella button loosening & $2(0.09 \%)$ & 0 & 0 \\
\hline Patello-femoral instability & $6(0.3 \%)$ & $1(0.5 \%)$ & 0 \\
\hline Patella fracture & $19(0.9 \%)$ & $1(0.5 \%)$ & $6(2 \%) *$ \\
\hline
\end{tabular}

BP index: Blackburne-Peel index; KSS: Knee Society Score; mFA: mechanical Femoral Axis; mFTA: mechanical Femoro-Tibial Axis; mTA: mechanical Tibial Axis

* significant difference $(\mathrm{p}<0.05)$ comparing the normal group

Table 3 Patello-femoral complications 
463 Table 4 Post-operative clinical and radiological characteristics of TKAs with normal pre-operative 464 patellar height $(\mathrm{N}=2248)$

465

\begin{tabular}{|c|c|c|c|}
\hline & $\begin{array}{c}\text { BP index }=0.54-1.06 \\
(N=1466)\end{array}$ & $\begin{array}{c}\text { BP index > } 1.06 \\
(N=57)\end{array}$ & $\begin{array}{c}\text { BP index }<0.54 \\
(N=725)\end{array}$ \\
\hline \multicolumn{4}{|l|}{ Range of motion } \\
\hline Mean flexion contracture & $0.5^{\circ}[0-20]$ & $1.3^{\circ}[0-40]$ & $0.5^{\circ}[0-25]$ \\
\hline Mean maximal flexion & $119^{\circ}[30-140]$ & $115.5^{\circ}[90-140]$ & $116.9^{\circ}[10-140]$ \\
\hline \multicolumn{4}{|l|}{ Mean KSS score } \\
\hline Knee & $88.3[24-100]$ & $88[45-100]$ & $88.2[19-100]$ \\
\hline Function & $78.1[0-100]$ & $74.7[0-100]$ & $78[0-100]$ \\
\hline \multicolumn{4}{|l|}{ Satisfaction } \\
\hline Very good and good & $1350(92.1 \%)$ & $51(89.5 \%)$ & $666(91.9 \%)$ \\
\hline Bad and very bad & $100(6.8 \%)$ & $4(7 \%)$ & $54(7.4 \%)$ \\
\hline$N C$ & $16(1.1 \%)$ & $2(3.5 \%)$ & $5(0.7 \%)$ \\
\hline \multicolumn{4}{|l|}{ Long leg films } \\
\hline Mean mFTA & $179^{\circ}[168-193]$ & $179^{\circ}[169-189]$ & $178.7^{\circ}[157-192]$ \\
\hline Mean $m F A$ & $89.6^{\circ}[78-98]$ & $89.4^{\circ}[83-94]$ & $89.3^{\circ}[77-98]$ \\
\hline Mean $m T A$ & $89.5^{\circ}[77-96]$ & $89.8^{\circ}[85-94]$ & $89.4^{\circ}[77-95]$ \\
\hline \multicolumn{4}{|l|}{ Complications } \\
\hline Stiffness & $31(2.1 \%)$ & $2(3.5 \%)$ & $15(2.1 \%)$ \\
\hline Clunk syndrome & $13(0.9 \%)$ & 0 & $5(0.7 \%)$ \\
\hline Extensor mechanism rupture & $3(0.2 \%)$ & 0 & $1(0.1 \%)$ \\
\hline Patella button loosening & $1(0.1 \%)$ & 0 & $1(0.1 \%)$ \\
\hline Patello-femoral instability & $5(0.3 \%)$ & 0 & $1(0.1 \%)$ \\
\hline Patella fracture & $11(0.8 \%)$ & $1(1.8 \%)$ & $7(1 \%)$ \\
\hline
\end{tabular}

466

467 BP index: Blackburne-Peel index; KSS: Knee Society Score; mFA: mechanical Femoral Axis; mFTA: 468 mechanical Femoro-Tibial Axis; mTA: mechanical Tibial Axis

$469 *$ significant difference $(\mathrm{p}<0.05)$ comparing the group with BP index $=0.54-1.06$ 
471 Table 5 Post-operative clinical and radiological characteristics of TKAs with pre-operative patella 472 alta $(\mathrm{N}=223)$

473

\begin{tabular}{|c|c|c|c|}
\hline & $\begin{array}{c}\text { BP index }=0.54-1.06 \\
(N=152)\end{array}$ & $\begin{array}{c}\text { BP index }>1.06 \\
(N=10)\end{array}$ & $\begin{array}{c}\text { BP index }<0.54 \\
(N=61)\end{array}$ \\
\hline \multicolumn{4}{|l|}{ Range of motion } \\
\hline Mean flexion contracture & $0.5^{\circ}[0-15]$ & $0.6^{\circ}[0-5]$ & $0.4^{\circ}[0-10]$ \\
\hline Mean maximal flexion & $120.3^{\circ}[85-140]$ & $114.5^{\circ}[35-135]$ & $115.4^{\circ}[20-140]$ \\
\hline \multicolumn{4}{|l|}{ Mean KSS score } \\
\hline Knee & $89.3[49-100]$ & $89.3[52-100]$ & $84.8[42-100] *$ \\
\hline Function & $79.9[20-100]$ & $83[45-100]$ & $81.3[40-100]$ \\
\hline \multicolumn{4}{|l|}{ Satisfaction } \\
\hline Very good and good & $140(92.1 \%)$ & $9(90 \%)$ & $55(90.2 \%)$ \\
\hline Bad and very bad & $12(7.9 \%)$ & 0 & $6(9.8 \%)$ \\
\hline$N C$ & 0 & $1(10 \%)$ & 0 \\
\hline \multicolumn{4}{|l|}{ Long leg films } \\
\hline Mean mFTA & $178.8^{\circ}[171-184]$ & $180^{\circ}[177-183]$ & $178.8^{\circ}[171-186]$ \\
\hline Mean $m F A$ & $89.3^{\circ}[83-94]$ & $90.3^{\circ}[88-92]$ & $90^{\circ}[82-96]$ \\
\hline Mean $m T A$ & $89.5^{\circ}[86-96]$ & $90^{\circ}[88-92]$ & $89.1^{\circ}[85-96]$ \\
\hline \multicolumn{4}{|l|}{ Complications } \\
\hline Stiffness & $1(0.7 \%)$ & 0 & $1(1.6 \%)$ \\
\hline Clunk syndrome & $1(0.7 \%)$ & 0 & $1(1.6 \%)$ \\
\hline Extensor mechanism rupture & 0 & 0 & 0 \\
\hline Patella button loosening & 0 & 0 & 0 \\
\hline Patello-femoral instability & $1(0.7 \%)$ & 0 & 0 \\
\hline Patella fracture & $1(0.7 \%)$ & 0 & 0 \\
\hline
\end{tabular}

474

475 BP index: Blackburne-Peel index; KSS: Knee Society Score; mFA: mechanical Femoral Axis; mFTA:

476 mechanical Femoro-Tibial Axis; mTA: mechanical Tibial Axis

$477 *$ significant difference $(\mathrm{p}<0.05)$ comparing the group with BP index $=0.54-1.06$ 
479 Table 6 Post-operative clinical and radiological characteristics of TKAs with pre-operative patella 480 baja $(\mathrm{N}=307)$

481

\begin{tabular}{|c|c|c|c|}
\hline & $\begin{array}{c}\text { BP index }=0.54-1.06 \\
(N=158)\end{array}$ & $\begin{array}{c}\text { BP index }>1.06 \\
(\mathrm{~N}=3)\end{array}$ & $\begin{array}{c}\text { BP index }<0.54 \\
(\mathrm{~N}=146)\end{array}$ \\
\hline \multicolumn{4}{|l|}{ Range of motion } \\
\hline Mean flexion contracture & $0.7^{\circ}[0-15]$ & $1.7^{\circ}[0-5]$ & $0.4^{\circ}[0-10]$ \\
\hline Mean maximal flexion & $118.4^{\circ}[70-140]$ & $116.7^{\circ}[85-105]$ & $112.8^{\circ}[70-140]$ \\
\hline \multicolumn{4}{|l|}{ Mean KSS score } \\
\hline Knee & $88.1[46-100]$ & $76.7[49-91]$ & $87.2[33-100]$ \\
\hline Function & $78.7[0-100]$ & $71.7[50-90]$ & $78.1[0-100]$ \\
\hline \multicolumn{4}{|l|}{ Satisfaction } \\
\hline Very good and good & $148(93.7 \%)$ & $2(67 \%)$ & $135(92.5 \%)$ \\
\hline Bad and very bad & $9(5.7 \%)$ & $1(33 \%)$ & $10(6.8 \%)$ \\
\hline$N C$ & $1(0.6 \%)$ & 0 & $1(0.7 \%)$ \\
\hline \multicolumn{4}{|l|}{ Long leg films } \\
\hline Mean mFTA & $178.8^{\circ}[171-184]$ & $179.3^{\circ}[178-180]$ & $177.9^{\circ}[168-185]$ \\
\hline Mean $m F A$ & $89.3^{\circ}[83-94]$ & $90^{\circ}[90-90]$ & $89^{\circ}[83-95]$ \\
\hline Mean $m T A$ & $89.5^{\circ}[86-96]$ & $89.3^{\circ}[88-90]$ & $89.2^{\circ}[83-95]$ \\
\hline \multicolumn{4}{|l|}{ Complications } \\
\hline Stiffness & $3(1.9 \%)$ & 0 & $8(5.5 \%) *$ \\
\hline Clunk syndrome & $2(1.3 \%)$ & 0 & $2(1.4 \%)$ \\
\hline Extensor mechanism rupture & 0 & 0 & 0 \\
\hline Patella button loosening & 0 & 0 & 0 \\
\hline Patello-femoral instability & 0 & 0 & 0 \\
\hline Patella fracture & $3(1.9 \%)$ & 0 & $3(2.1 \%)$ \\
\hline
\end{tabular}

482

483 BP index: Blackburne-Peel index; KSS: Knee Society Score; mFA: mechanical Femoral Axis; mFTA:

484 mechanical Femoro-Tibial Axis; mTA: mechanical Tibial Axis

$485 *$ significant difference $(\mathrm{p}<0.05)$ comparing the group with BP index $=0.54-1.06$ 
487 Table 7 Specific results of patients with tibial tubercle osteotomy $(\mathrm{N}=128)$

488

489

\begin{tabular}{|c|c|c|c|}
\hline & $\begin{array}{c}\text { Pre-operative } \\
\text { BP index }=0.54-1.06 \\
(\mathrm{~N}=101)\end{array}$ & $\begin{array}{c}\text { Pre-operative } \\
\text { BP index }>1.06 \\
(N=12)\end{array}$ & $\begin{array}{c}\text { Pre-operative } \\
\text { BP index }<0.54 \\
(N=15)\end{array}$ \\
\hline \multicolumn{4}{|l|}{ Medical history } \\
\hline Lateral approach for valgus & $90(89.1 \%)$ & $11(91.7 \%)$ & $10(66.7 \%) *$ \\
\hline Stiffness & $11(10.9 \%)$ & $1(8.3 \%)$ & $5(33.3 \%) *$ \\
\hline \multicolumn{4}{|l|}{ Post-operative BP index } \\
\hline$B P$ index $=0.54-1.06$ & $69(68.3 \%)$ & $8(66.7 \%)$ & $8(53.3 \%)$ \\
\hline$B P$ index $>1.06$ & $3(3 \%)$ & $1(8.3 \%)$ & 0 \\
\hline$B P$ index $<0.54$ & $29(28.7 \%)$ & $3(25 \%)$ & $7(46.7 \%) *$ \\
\hline
\end{tabular}

490 BP index: Blackburne-Peel index

$491 *$ significant difference $(\mathrm{p}<0.05)$ comparing the group with BP index $=0.54-1.06$

492 
494 FIGURES

495

496

497 Fig. 1 Blackburne-Peel index $=\mathrm{A} / \mathrm{B}$ (profile view)

498

a: on native knee

499

b: on TKA

500

501

502 Fig. 2 Native knee with normal patellar height (profile view)

503

504

505 Fig. 3 Native knee with patella alta (profile view)

506

507

508 Fig. 4 Native knee with patella baja (profile view)

509

510

511 Fig. 5 Flowchart

512

513

514 Fig. 6 Survival curves of TKA

515

a: with pre-operative normal patellar height

516

b: with pre-operative patella alta

517

c: with pre-operative patella baja

518 


$$
\sum_{\lambda}
$$




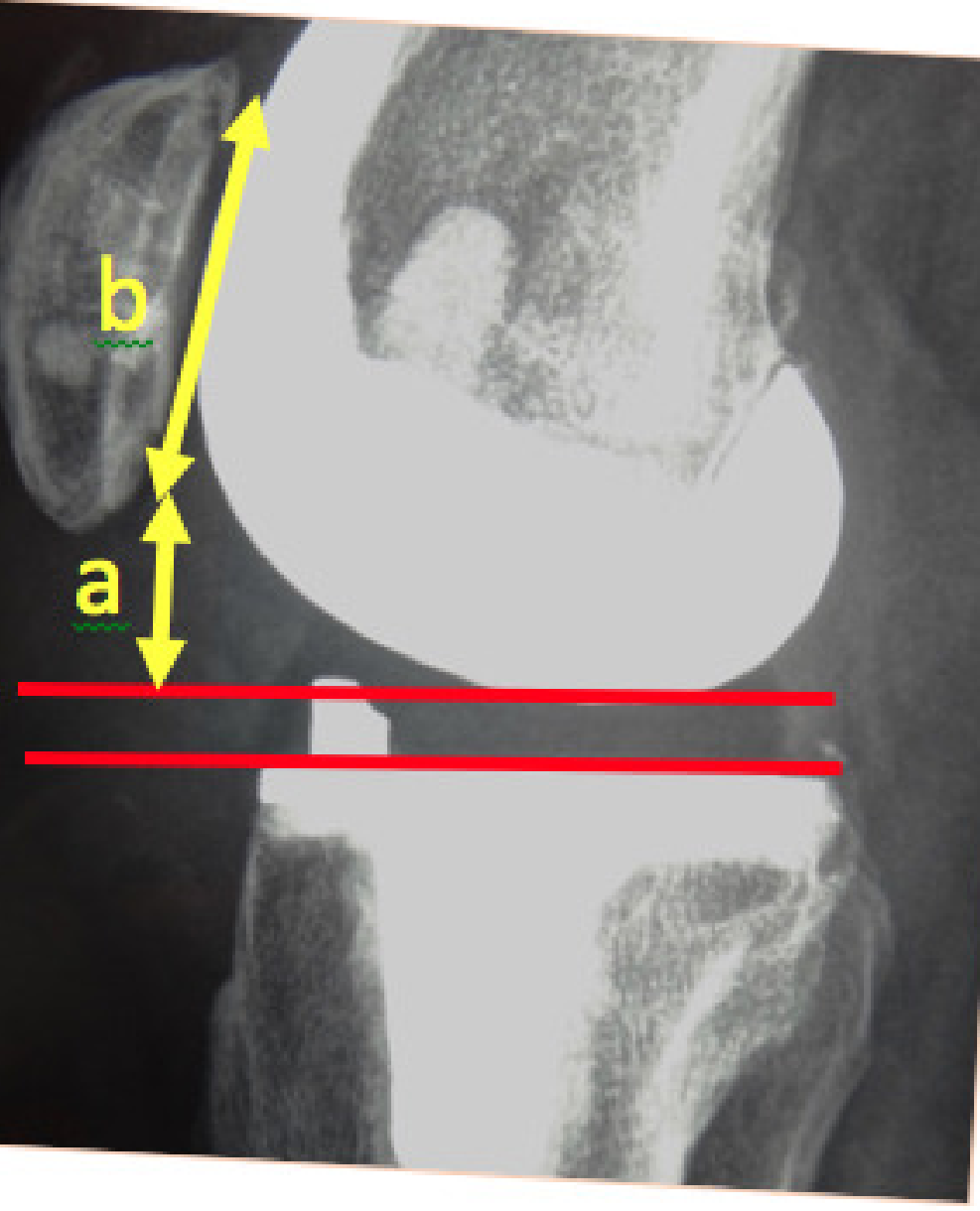




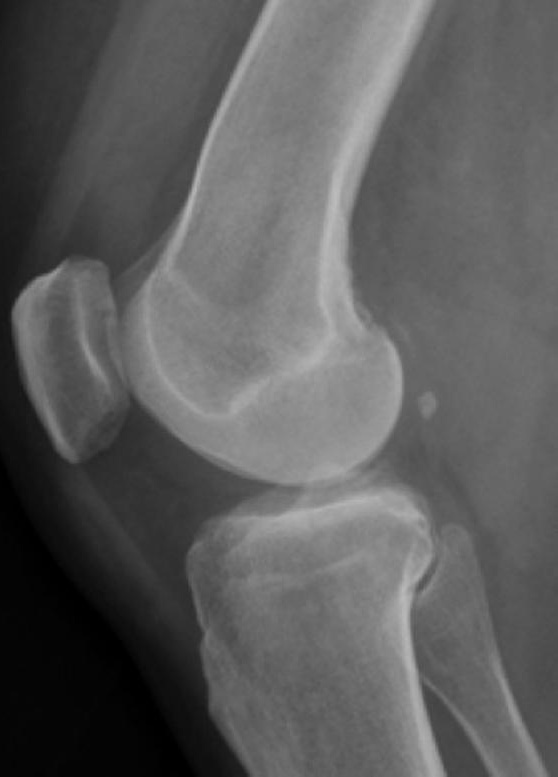




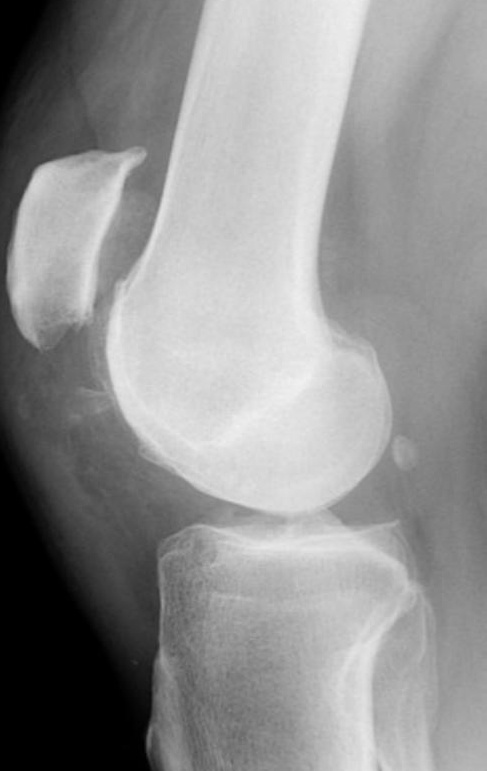




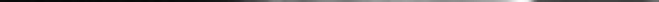


Initial cohort

$$
\mathrm{n}=4103
$$

Normal patellar height

$$
\mathrm{n}=3301
$$

Patella alta

$$
\mathrm{n}=330
$$

\section{Patella baja}

$$
\mathrm{n}=472
$$

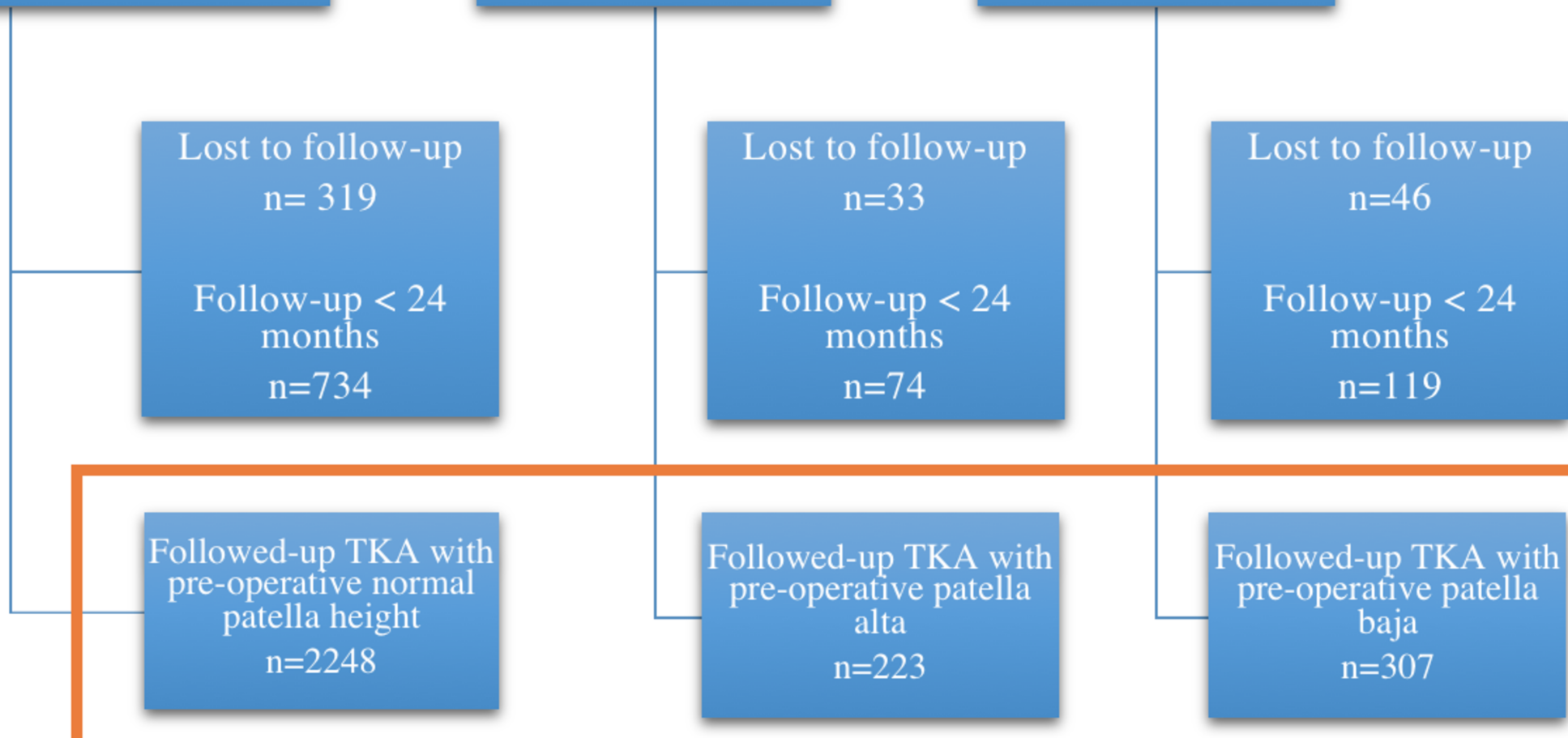

\section{Pre- and post-operative analysis}


« Normal patellar height group » $(\mathrm{N}=\mathbf{2 2 4 8})$

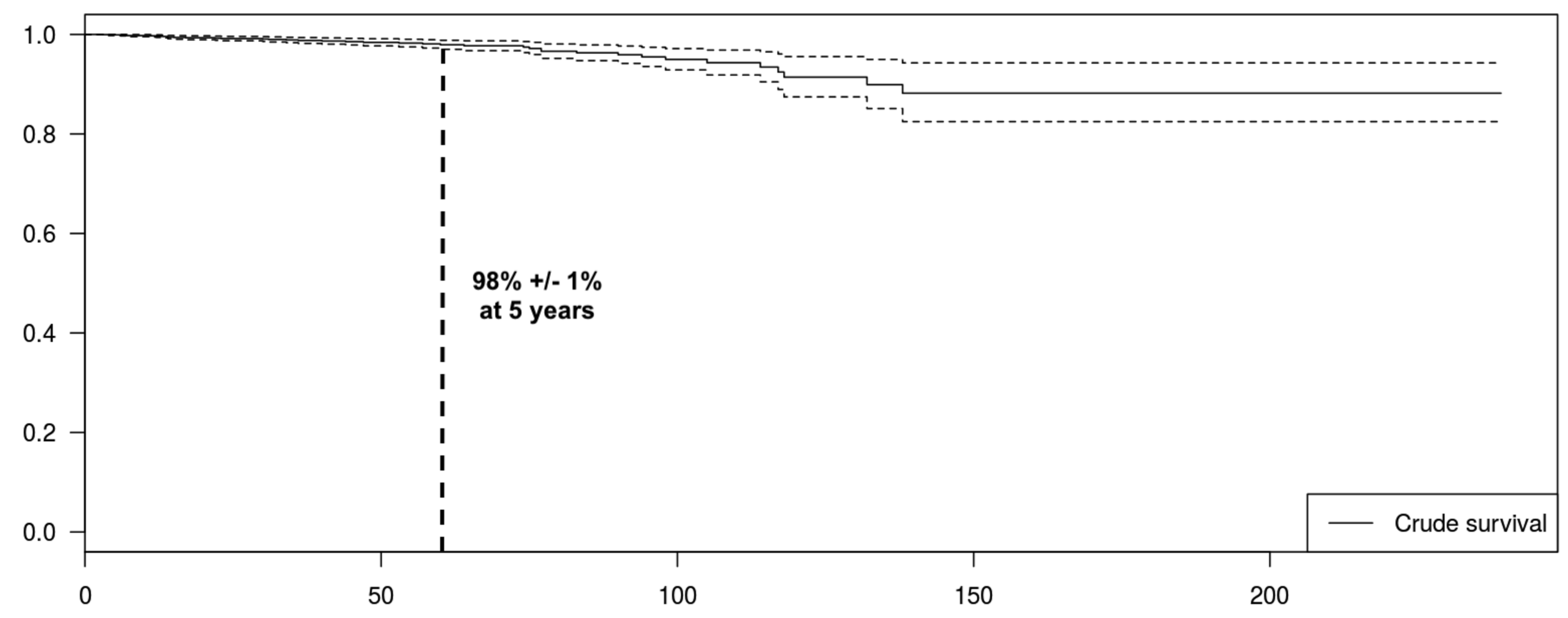


« Patella alta group » $(\mathrm{N}=223)$

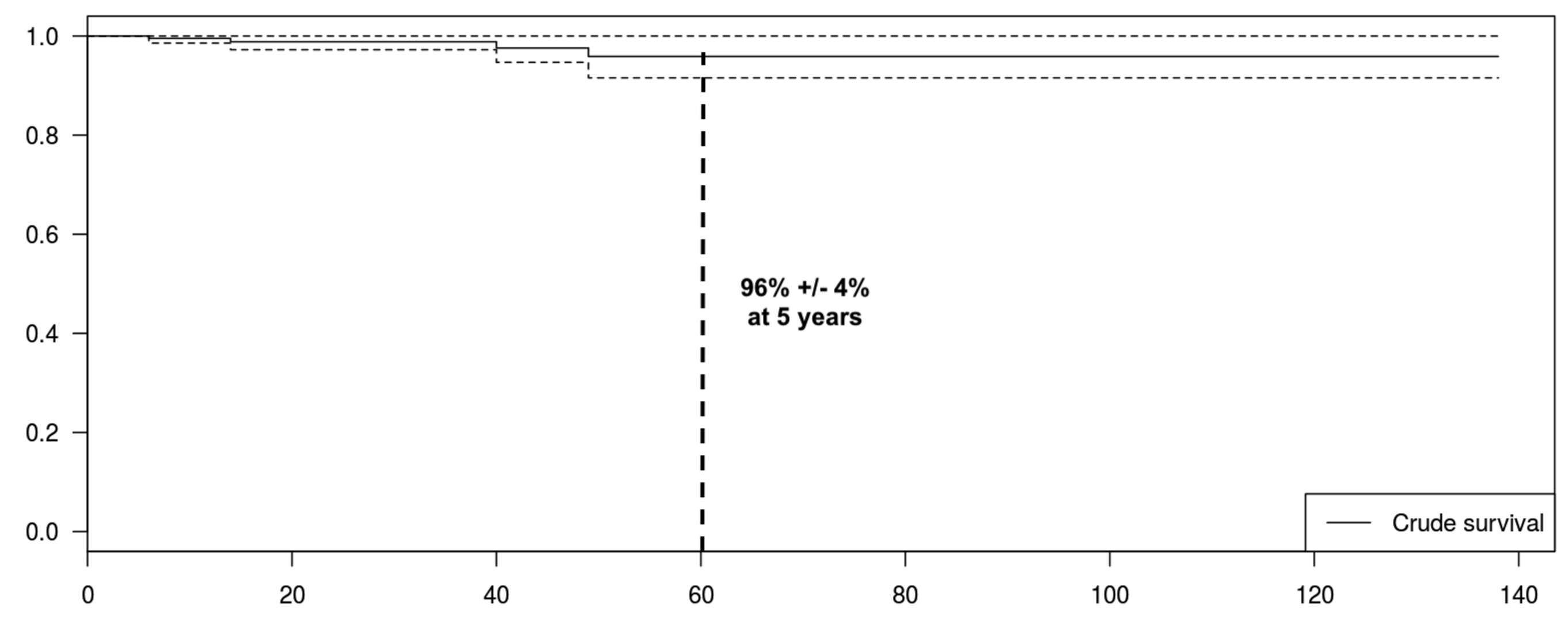


«Patella baja group » $(\mathrm{N}=\mathbf{3 0 7})$

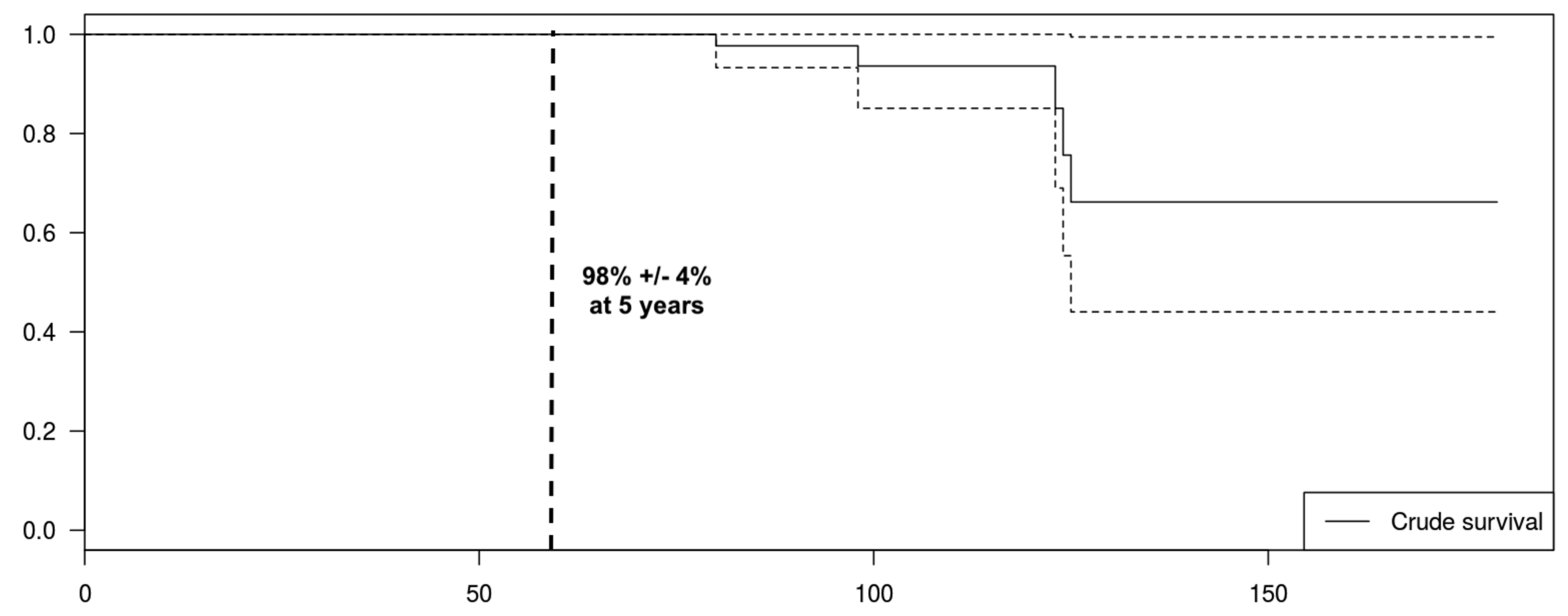

